\title{
Liver Neuroendocrine Tumor
}

National Cancer Institute

\section{Source}

National Cancer Institute. Liver Neuroendocrine Tumor. NCI Thesaurus. Code C5783.

An extremely rare, usually solitary, well-differentiated neuroendocrine tumor (carcinoid

tumor) arising from the liver. There is mild nuclear atypia and low mitotic activity present.

The neoplastic cells express immunohistochemical evidence of neuroendocrine

differentiation. The vast majority of carcinoid tumors found in the liver represent metastases from other anatomic sites. 